\title{
Diabetes care in Singapore: how an integrated approach can help meet the challenges ahead
}

\author{
Joo Pin Foo $^{1}$, MBBS, MRCP, Kwong Ming Fock ${ }^{2}$, MBBS, FRCP
}

T he increasing prevalence of diabetes mellitus and its consequent burden on healthcare resources is a major concern in many countries around the world. Worldwide, the number of people with diabetes mellitus has increased from 153 million in 1980 to 347 million in 2008, ${ }^{(1)}$ and is projected to increase to 552 million by 2030. ${ }^{(2)}$ Singapore will not be spared from this 'diabetes tsunami'. In Singapore, the prevalence of diabetes mellitus among adults aged $18-69$ years has increased from $8.2 \%$ of the population in the year 2004 to $11.3 \%$ in $2010 .{ }^{(3)}$ The rapidly aging population, coupled with the increasing prevalence of obesity, looks set to aggravate the numbers. The burden of diabetes mellitus lies not only in the treatment of hyperglycaemia, but more so in the associated micro- and macrovascular complications that may lead to visual impairment, amputations and renal failure.

It has been well demonstrated in the United Kingdom Prospective Diabetes Study (UKPDS) and the Diabetes Control and Complication Trial (DCCT) that treatments which target glycated haemoglobin (HbA1c) levels significantly reduce the risk of diabetic micro- and macrovascular complications. ${ }^{(4,5)}$ The principle of achieving target $\mathrm{HbA1c}$ level, which is clear to most medical practitioners, has guided diabetes care in the last few decades. However, in practice, finding ways to further increase the proportion of patients who achieve and maintain their target $\mathrm{HbA} 1 \mathrm{c}$ levels remains a challenge. With the abundance of healthcare services and primary care in Singapore, the major factor in determining diabetes mellitus outcome is thus not access to care, but rather, improvement in the quality of diabetes care.

\section{CHALLENGES IN DIABETES CARE}

Obstacles to the provision of quality diabetes care are complex and multifactorial. Complete diabetic evaluation and management should include efforts to classify diabetes mellitus precisely, screen for diabetes complications, review risk factor control, adopt an appropriate treatment regimen and provide continued care. ${ }^{(6)}$ Clinical practice guidelines (CPG) have served to update many practitioners and provide recommendations on the standards of care for many chronic diseases. Continuing medical education (CME) has provided multiple channels for primary care physicians to be updated on current practices in diabetes mellitus. Despite these guidelines and programmes, differing practices with varying standards of care still exist in the medical community. These varying standards of care may lead to late diagnosis, failure to attain target $\mathrm{HbA} 1 \mathrm{c}$, and delay in the detection of complications that may have benefited from earlier intervention. There is therefore a need for a concerted effort to define what constitutes a minimum standard of diabetes care, and more importantly, a mechanism to monitor whether these standards have been adhered to in a consistent manner.

While the abundance of primary and specialist care services from both public and private sectors means that the community is well served and has easy access to medical care, the downside may be duplication of tests, wastage of resources, and a lack of coordinated care between various primary care physicians and specialists. This will ultimately lead to confusion among patients, who may then default on some appointments or treatments that are imperative in their condition. Moreover, the current incoordination of care does not allow for the practice of stratified medicine in which treatments (including pharmacological and non-pharmacological interventions) are targeted based on the biological or risk characteristics of a group of patients. In view of the current situation, there is a need for collaboration within the multidisciplinary care team, and perhaps a mechanism to oversee the various aspects of diabetes care, so as to ensure that patients' risk profiles are better stratified, care is targeted and streamlined with minimal duplication, and an optimal standard of care is achieved.

While the key to successful diabetes management lies in appropriate and coordinated care from healthcare providers, there is an underappreciation of the role of patient education. Various authorities, including the American Diabetes Association (ADA), National Institute for Health and Care Excellence (NICE) and the Scottish Intercollegiate Guidelines Network (SIGN), have issued guidelines that emphasise the importance of patient education and self-management skills, ${ }^{(67)}$ without which a higher rate of treatment failure will most certainly result. Knowledge and skills, such as compliance to medication, dietary adherence and lifestyle modifications, are imperative to the successful management of diabetes mellitus. Other important skills, especially among insulin-treated patients, include insulin injection techniques, self-monitoring of blood glucose (SMBG), self-titration of insulin, avoidance and self-management of hypoglycaemia, sick day rules and carbohydrates counting. Encouraging patients to conduct self-monitoring, foot checks,

${ }^{1}$ Department of Endocrinology, ${ }^{2}$ Department of Medicine, Changi General Hospital, Singapore.

Correspondence: Dr Foo Joo Pin, Consultant and Director, Diabetes Centre, Changi General Hospital, 2 Simei Street 3 , Singapore 529889. joo_pin_foo@cgh.com.sg 
interventions such as smoking cessation, and management of comorbidities such as hypertension and hyperlipidaemia, are important measures that cannot be ignored. Such knowledge or instruction may be imparted by the doctor or more often, in the case of a tertiary centre or polyclinic, by a diabetic nurse educator (DNE).

Not uncommonly, many diabetic patients still lack important self-management skills despite multiple efforts at education. Often, this results in failure to achieve target HbA1c levels, and could even result in hospitalisation for diabetes-related complications. Notwithstanding the fact that the layperson may have difficulties understanding the instructions given, patient education often takes place during hospital admission for a diabetes-related condition, when the patient may not be in a conducive state to retain such information, thus possibly contributing to the failure of the patient to fully grasp the essence of the instruction. Therefore, besides stepping up patient education efforts by increasing the number of counselling sessions, a systematic way of educating the patients, which may not take place during admission for an acute illness and which allows for reinforcement and feedback, needs to be in place to ensure that patients are better educated with these important self-management skills.

The failure of patients to adequately control their diabetes mellitus can be attributed to a plethora of reasons. One of these may be technical issues with the administration of medication; for example, a patient with retinopathy and poor visual acuity may face difficulties drawing insulin from a vial and performing self-injection. Other reasons could be a lack of social support or care at home, the patient's beliefs and attitudes, financial constraints, comorbidities, lack of understanding leading to poor compliance, or psychological issues. ${ }^{(8)}$ While clinicians are aware that such barriers, coupled with inadequate intervention strategies, may have resulted in their patient's poor glycaemic control, they still face the monumental task of identifying these barriers within the short consultation period with each patient. It is even more challenging to institute solutions that effectively address all these barriers within the limited time. Moreover, the majority of these issues unfold in the patient's home, which the doctor has no access to or has limited knowledge of. Hence, these issues may not be detected during the consultation.

In view of the aforementioned challenges, a system that acts as an extension of the doctor's consultation and enables monitoring of the patient's condition in his/her home should be put in place. This will not only allow for the detection of barriers to successful diabetes care that may otherwise be missed during regular consultation, but also serves as a second line of support between doctor's visits. When such a support and monitoring system is established, the service may then be expanded to provide timely intervention. For example, patients who have persistent hyperglycaemia (due to inadequate control despite recent dose increment) or hypoglycaemia (as a result of medication changes) do not have to wait until the next doctor's visit before the issue is detected and addressed. The monitoring system should incorporate an activation pathway so that patients with complications that may result in hospitalisation if not addressed quickly can receive timely recommendations and intervention from DNEs or other members of the diabetic multidisciplinary team. In this way, the issue can be resolved the moment it is identified, without the patients having to wait until the next consultation.

\section{THE INTEGRATED APPROACH}

To address the current gaps in diabetes care in the country, it is important to put in place a system that enables patients' education to be intensified in a conducive environment, incorporates monitoring with appropriate intervention and facilitates an overview of patients' multiple medical, logistic and administrative issues, and thereafter, to streamline care and reinforce consistent practice while adhering to the current standards of diabetes care. Once such a system is well established, the clinical data and indicators collected through the system can be further utilised for risk stratification and targeting of treatment, based on the individual patient's risk profile.

In 2010, the Health Management Unit (HMU) was set up in Changi General Hospital $(\mathrm{CGH})$ to fulfill the abovementioned role (Fig. 1). HMU is a telehealth service supported by a Patient Relationship Management (PRM) IT system, which is central to the programme. The PRM IT system enables tele-nurses to access relevant clinical indicators and information, as well as capture all telephone interactions with the patient. Patients who have been treated for diabetes mellitus within the $\mathrm{CGH}$ healthcare system and who fulfill the inclusion criteria are recruited into the programme based on their International Classification of Diseases (ICD) codes. The patients are contacted by telenurses during office hours, although provisions are made to accommodate patients who are only available after office hours. The system is inbuilt with scripts that cover various aspects of diabetes education, including dietary advice, lifestyle modification, hypoglycaemia management, foot care advice, smoking cessation and management of comorbidities. The scripts were prepared using evidence-based guidelines, including international guidelines of ADA and local CPG. Feedback from specialist doctors and allied health professionals was also sought during the preparation of the scripts. The topics of the script are covered in a systematic way, which allows for feedback in order to check that the patient understands, through a series of scheduled telephone calls to the patient. Apart from patient education, other components within the programme include encouragement of self-monitoring, recording of such monitoring, coaching to overcome barriers, collection and monitoring of clinical markers of the patient, and care coordination.

Through this well-scripted protocol, not only do the patients receive instructions in the comfort of their own homes, the interactions also allow problems and barriers faced by patients, which would usually not be apparent to the physician during 


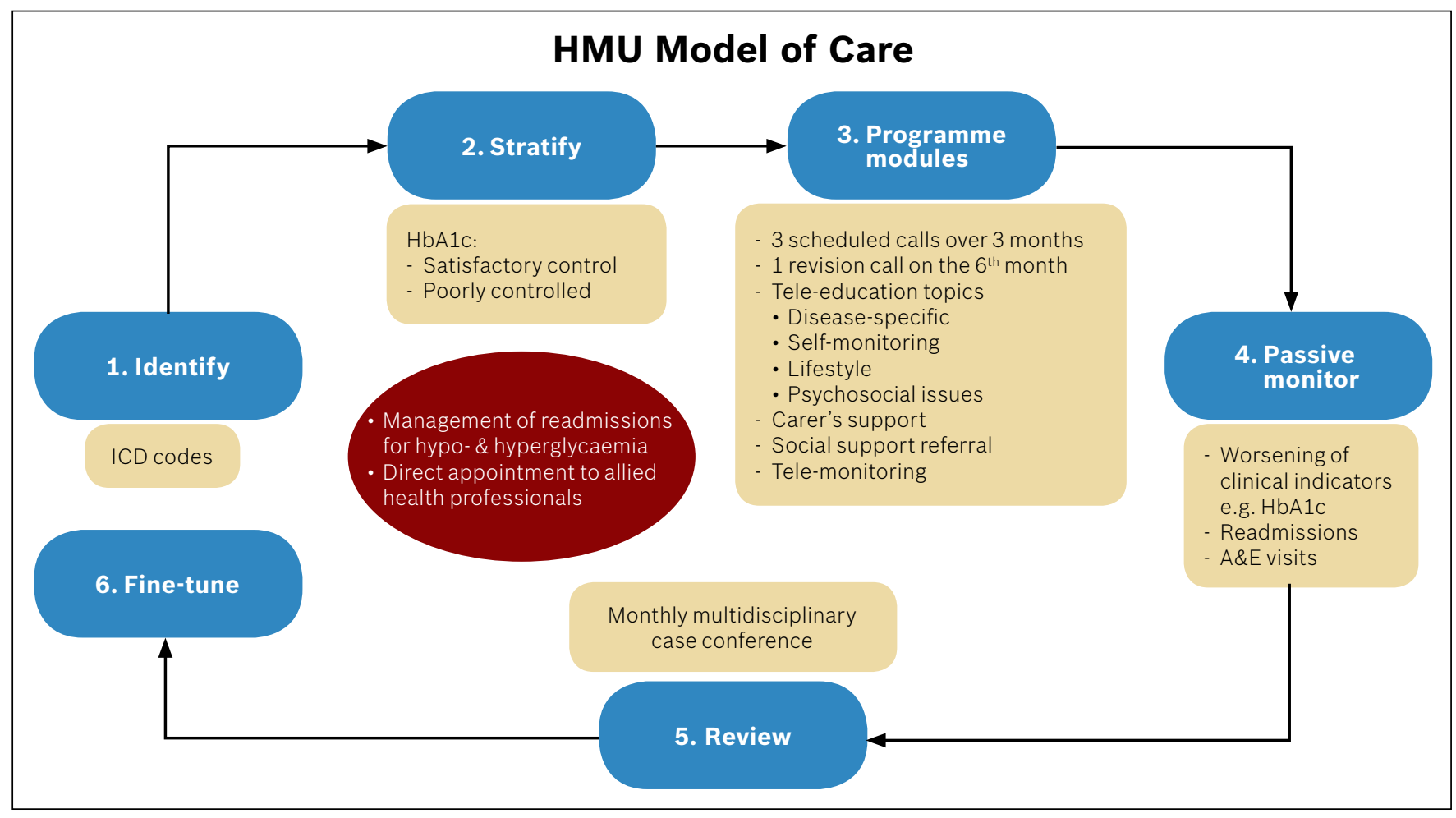

Fig. 1 Schematic diagram shows the workflow and components of the model of care utilised by the Health Management Unit.

A\&E: accident and emergency; HbA1c: glycated haemoglobin; HMU:Health Management Unit; ICD: International Classicfication of Diseases

the short consultations, to be identified immediately. Upon the identification of a problem, a series of pathways can be activated to bring about timely and appropriate intervention. For example, a patient who is identified by a tele-nurse to have psychosocial issues resulting in poor care will be referred to medical social workers and psychologists, who will then render the appropriate intervention. Another example would be a patient on insulin who suffers from hypoglycaemia at home - once detected by the tele-nurse, the patient will be escalated to the DNE, who will then advise the patient on hypoglycaemia management and insulin titration before the episode worsens and results in hospitalisation. Various activation pathways have been built in the system, including escalation to dietitians, clinical psychologists, medical social workers, action teams, DNEs, and even smoking cessation clinics, all of which support the patient and provide timely intervention between doctor's visits.

The system auto-extracts and condenses the patient's information (e.g. capillary glucose readings at home, appointments with the diabetes multidisciplinary team, important clinical indicators such as $\mathrm{HbA1c}$ and lipids profile), together with the problems (e.g. dietary and medication compliance, social or psychological issues) identified during the interaction between the patient and tele-nurse, into an easy-to-read format for the principal doctor's reference during consultation. Additionally, a multidisciplinary case conference is held every month to discuss specific cases with worsening clinical indicators and frequent readmissions for diabetes-related conditions despite HMU education and support. This allows for the mobilisation of appropriate interventions to address issues precipitating these readmissions. All these measures lead to better coordinated care and enable managing physicians to be well informed of the issues plaguing the patients with various problems, which may well be resolved even before the doctor's visit, thereby optimising patient care.

It must be emphasised, however, that the role of a telehealth service like HMU is not meant to supersede or circumvent the usual care and existing treatment that patients are receiving from their primary physicians or allied health professionals. The service was developed to play a supportive role in patient education and provide encouragement of self-monitoring, coaching to overcome barriers, and monitoring of complications and care coordination, on top of the existing care that patients are already receiving. Appropriate intervention, if necessary, will be escalated and referred to relevant allied health professionals, such as DNEs or the patient's primary physician, for further action or attention. Treatment decisions made by the primary physician or allied health professional are strictly adhered to, and the advice given by the tele-nurses is nonintrusive and noncontradictory to the advice the patients have received from their physicians or allied health professionals. During the interaction between the tele-nurse and patient, it is emphasised to the patients that the support provided by the tele-nurse is only for disease-specific management and not meant to address other complaints. Issues unrelated to disease-specific management that may arise during the interaction will be responded to professionally.

The design of the programme also took into consideration the social fabric and cultural context of the local population. For example, in the local Asian context, where much of the care decision regarding the patient lies with the family members 
(especially grown children), protocols are instituted in which the carers are identified, educated and supported in addition to the patient. Tele-nurses have also been trained to speak in the preferred language of patients or carers, and these languages include all local dialects and languages spoken by the various ethnic groups in Singapore.

\section{CONCLUSION}

Diabetes care requires a multidisciplinary approach via a coordinated effort to bring together the various support services in a timely and effective manner. Different models of telehealth disease management programmes have been demonstrated to reduce hospital admissions and improve diabetes care in many countries. ${ }^{(9-11)}$ The telehealth service is a novel model of care in Singapore, and is possibly underutilised in view of the high penetration of telecommunication devices in the country. Telehealth service, coupled with an advanced IT system, has the potential to fill many of the existing gaps as well as improve the quality of diabetes care in Singapore. The challenge lies in how to further adapt this evolving model of care to the cultural background and social circumstances specific to the local setting, so that its maximum potential can be realised. It remains to be determined how such a telehealth service can impact diabetes outcome in the long term and whether it can be justified based on cost effectiveness. Given the current challenges facing diabetes care in the country, an integrated approach utilising such a system might just be the answer to the problems.

\section{REFERENCES}

1. Danaei G, Finucane MM, Lu Y, et al. National, regional, and global trends in fasting plasma glucose and diabetes prevalence since 1980: systematic analysis of health examination surveys and epidemiological studies with 370 country-years and 2.7 million participants. Lancet 2011; 378:31-40.

2. Whiting DR, Guariguata L, Weil C, Shaw J. IDF diabetes atlas: global estimates of the prevalence of diabetes for 2011 and 2030. Diabetes Res Clin Prac 2011; 94:311-21.

3. National Health Survey 2004. Singapore: Epidemiology and Disease Control Division, Ministry of Health, 2010.

4. Holman RR, Paul SK, Bethel MA, Matthews DR, Neil HA. 10-year follow-up of intensive glucose control in type 2 diabetes. N Eng J Med 2008; 359:1577-89.

5. The effect of intensive treatment of diabetes on the development and progression of long-term complications in insulin-dependent diabetes mellitus. The Diabetes Control and Complications Trial Research Group. N Eng J Med 1993; 329:977-86.

6. American Diabetes Association. Standards of medical care in diabetes 2013. Diabetes Care 2013; 36 Suppl 1:S11-66.

7. Type 2 Diabetes: National Clinical Guideline for Management in Primary and Secondary Care (Update). National Institute for Health and Clinical Excellence Clinical Guidelines, No. 66. London: Royal College of Physicians (UK), 2008.

8. Nam S, Chesla C, Stotts NA, Kroon L, Janson SL. Barriers to diabetes management: patient and provider factors. Diabetes Res Clin Pract 2011; 93:1-9.

9. Young RJ, Taylor J, Friede T, et al. Pro-active call center treatment support (PACCTS) to improve glucose control in type 2 diabetes: a randomized controlled trial. Diabetes care 2005; 28:278-82.

10. Steventon A, Bardsley M, Billings J, et al. Effect of telehealth on use of secondary care and mortality: findings from the Whole System Demonstrator cluster randomised trial. BMJ 2012; 344:e3874.

11. Weinstock RS, Teresi JA, Goland R, et al. Glycemic control and health disparities in older ethnically diverse underserved adults with diabetes: five-year results from the Informatics for Diabetes Education and Telemedicine (IDEATel) study. Diabetes care 2011; 34:274-9.

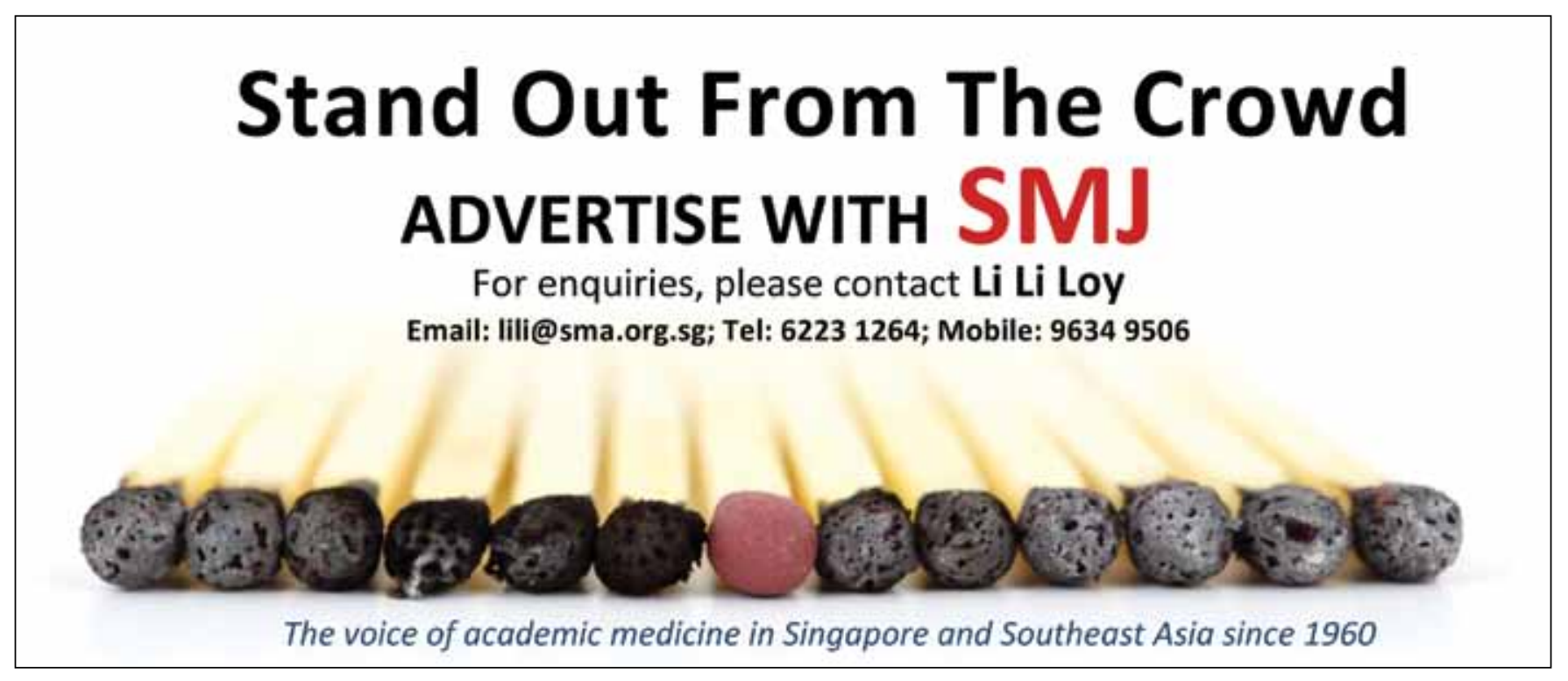

